\title{
DIRECT OBSERVATIONS OF SUBMICROPULSE ELECTRON-BEAM EFFECTS FROM SHORT-RANGE WAKEFIELDS IN TESLA-TYPE SUPERCONDUCTING RF CAVITIES *
}

\author{
A. H. Lumpkin†, R. M. Thurman-Keup, D. R. Edstrom Jr., P. Prieto, J. Ruan, \\ Fermi National Accelerator Laboratory, Batavia, IL, USA \\ B. T. Jacobson, A. L. Edelen, J. A. Diaz-Cruz, F. Zhou, \\ SLAC National Accelerator Laboratory, Menlo Park, CA, USA
}

\section{Abstract}

Experiments were performed at The Fermilab Accelerator Science and Technology (FAST) facility to elucidate the effects of short-range wakefields (SRWs) in TESLAtype rf cavities. FAST has a unique configuration of a photocathode rf gun beam injecting two TESLA-type single cavities (CC1 and $\mathrm{CC} 2)$ in series prior to the cryomodule. To investigate short-range wakefield effects, we have steered the beam to minimize the signals in the higher-order mode (HOM) detectors of $\mathrm{CC} 1$ and $\mathrm{CC} 2$ for a baseline, and then used a vertical corrector between the two cavities to steer the beam off axis at an angle into CC2. A Hamamatsu synchroscan streak camera viewing a downstream OTR screen provided an image of y-t effects within the micropulses with $\sim 10$-micron spatial resolution and 2-ps temporal resolution. At $500 \mathrm{pC} / \mathrm{b}, 50 \mathrm{~b}$, and $4 \mathrm{mrad}$ off-axis steering into $\mathrm{CC} 2$, we observed an $\sim 100$-micron head-tail centroid shift in the streak camera image $\mathrm{y}(\mathrm{t})$-profiles. This centroid shift value is 5 times larger than the observed HOM-driven centroid oscillation within the macropulse, and it is consistent with a calculat$\dot{\sigma}$ ed short-range wakefield effect using ASTRA simulaते tions.

\section{SUBMISSION OF PAPERS}

The preservation of the low emittance of electron beams during transport through the accelerating structures of large facilities is an ongoing challenge. In the cases of ${ }^{\circ}$ the TESLA-type superconducting rf cavities currently $\checkmark$ used in the European X-ray Free-electron Laser (FEL) [1] and the currently-under-construction Linac Coherent $\checkmark$ Light Source upgrade (LCLS-II XFEL) [2], off-axis beam transport may result in emittance dilution due to transv verse long-range (LRW) and short-range wakefields (SRW) [3-5]. To investigate such effects, experiments J were performed at the Fermilab Accelerator Science and Technology (FAST) facility with its unique two-cavity configuration after the photocathode rf gun [6]. We used optical transition radiation (OTR) imaging with a UVvisible synchroscan streak camera to display submicropulse $y$-t effects in the $41-\mathrm{MeV}$ beam.

We report effects on beam transverse position centroids and sizes correlated with off-axis beam steering in TESLA-type cavities. We used a 3-MHz micropulse repetition

* This manuscript has been authored by Fermi Research Alliance, LLC under Contract No. DE-AC02-07CH11359 with the U.S. Department of Energy, Office of Science, Office of High Energy Physics. † lumpkin@fnal.gov

rate and targeted diagnostics for these tests. Our initial data from an OTR imaging source indicated our streak camera can provide $\sim 10$-micron spatial resolution with $1-2$ ps $(\sigma)$ temporal resolution depending on the bandpass filter employed. Since the observed bunch lengths were $10-20$ ps $(\sigma)$, we had sufficient resolution for up to 20 time slices in the $4 \sigma$ profile. In this sense we also obtained slice-emittance information (with $\beta$-function information). We used the higher-order mode (HOM) detectors and rf BPMs to establish first the desired off-axis steering and then evaluated the short-range wakefield effects on the beam dynamics.

\section{EXPERIMENTAL ASPECTS}

\section{The IOTA Electron Injector Linac}

The Integrable Optics Test Accelerator (IOTA) electron injector at the FAST facility (Fig. 1) begins with an Lband rf photoinjector gun built around a Cs2Te photocathode (PC). When the UV component of the drive laser, described elsewhere [7] is incident on the PC, the resulting electron bunch train with $3-\mathrm{MHz}$ micropulse repetition rate exits the gun at $<5 \mathrm{MeV}$. Following a short transport section with a pair of trim dipole magnet packages (H/V100 and H/V101), the beam passes through two superconducting rf (SCRF) capture cavities denoted CC1 and $\mathrm{CC} 2$, and then a transport section to the low-energy electron spectrometer, D122. Diagnostics used in these studies include the rf BPMs, the imaging screens at X107, $\mathrm{X} 108, \mathrm{X} 121$, and X124, and HOM couplers at the upstream and downstream ends of each SCRF cavity. The HOM signals were processed by the HOM detector circuits with the Schottky diode output provided online though ACNET, the Fermilab accelerator controls network [4]. The HOM detectors' bandpass filters were optimized for two dipole passbands from 1.6 to $1.9 \mathrm{GHz}$, and the $1.3-\mathrm{GHz}$ fundamental was reduced with a notch filter. The rf BPMs' electronics were configured for bunch-by-bunch capability with optimized system attenuation. At $2 \mathrm{nC}$ per micropulse, the rms noise was found to be $25 \mu \mathrm{m}$ in the horizontal axis (x) and $15 \mu \mathrm{m}$ in the vertical axis (y) at B101 in the test with $4.5-\mathrm{MeV}$ beam from the gun. However, for these studies on short-range transverse wakefields, we relied on a streak camera to provide the sub-micropulse spatial information. 


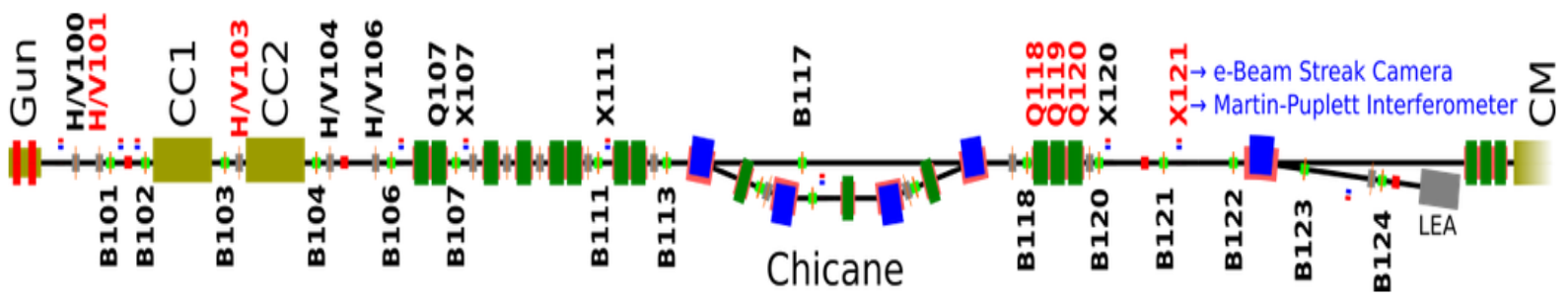

Figure 1: Schematic of the FAST beamline layout showing the PC rf gun, capture cavities $(\mathrm{CCn})$, correctors (H/Vnnn), rf BPMs (Bnnn), chicane, OTR and YAG:Ce screens (Xnnn), the spectrometer dipole bend magnet (D122), and the beginning of the cryomodule $(\mathrm{CM})$.

\section{The Streak Camera System}

We utilized a C5680 Hamamatsu streak camera with an S20 PC operating with the M5675 synchroscan vertical deflection unit that was phase locked to $81.25 \mathrm{MHz}$ as shown in Fig. 2. In addition, we used a phase-locked-loop C6878 delay box that stabilizes the streak image positions to about 1 ps temporal jitter over $10 \mathrm{~s}$ of minutes. These steps enabled the synchronous summing of 50-150 micropulses or bunches (b) generated at $3 \mathrm{MHz}$ by the photoinjector or the offline summing of 10-100 images to improve statistics in the sum images. We applied the principle to optical transition radiation (OTR) generated from an Al-coated Sisubstrate at the X121 screen location (see Fig. 1) with subsequent transport to the beamline streak camera. Commissioning of the streak camera system was facilitated through a suite of controls centered around ACNET. This suite includes operational drivers to control and monitor the streak camera as well as Synoptic displays to facilitate interface with the driver. Images were captured from the streak camera using the readout camera, Prosilica 1.3-Mpixel cameras with $2 / 3$ " format, and were analyzed both online and with an offline MATLABbased ImageTool processing program [8]. Bunch-length measurements using these techniques have been reported previously from the A0 Facility [9] and FAST first system streak camera commissioning at $20 \mathrm{MeV}$ [10].

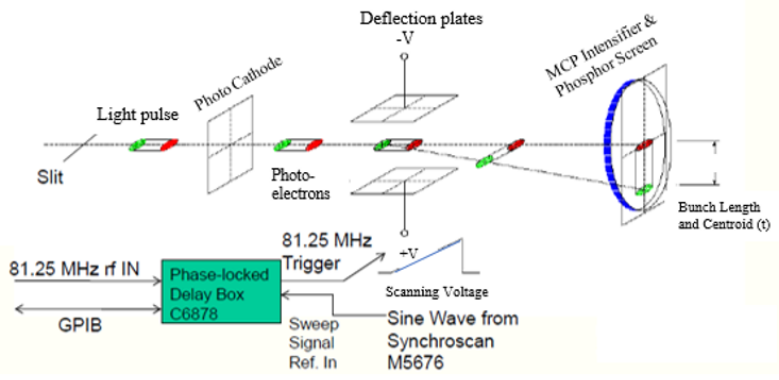

Figure 2: Schematic of the C5680 synchroscan streak camera with phase locking at $81.25 \mathrm{MHz}$.

\section{EXPERIMENTAL RESULTS}

\section{Initial Streak Camera Data}

In order to investigate the short-range, wakefield-driven submicropulse effects, we used the HOM detector signals as a measure of how far off axis the beam was in the cavities. We minimized the HOMs in $\mathrm{CC} 1$ and $\mathrm{CC} 2$ as the reference point, and then stepped the V103 corrector magnet current values. For the $24-\mathrm{MeV}$ post-CC1 beam energy, a change of $1 \mathrm{~A}$ in corrector current corresponded to a 2-mrad angular steering change into $\mathrm{CC} 2$. The transport optics rotated the image $90^{\circ}$ so we observed the vertical spatial information on the horizontal display axis in the streak image from X121. The changes in projected vertical beam profile size are shown in Fig. 3a. Beam size dilution is clearly seen at $650 \mu \mathrm{m}$ compared to the $400-\mu \mathrm{m}$ cases. In Fig. 3b, the head-tail kick directions follow the corrector steering polarity as expected. These
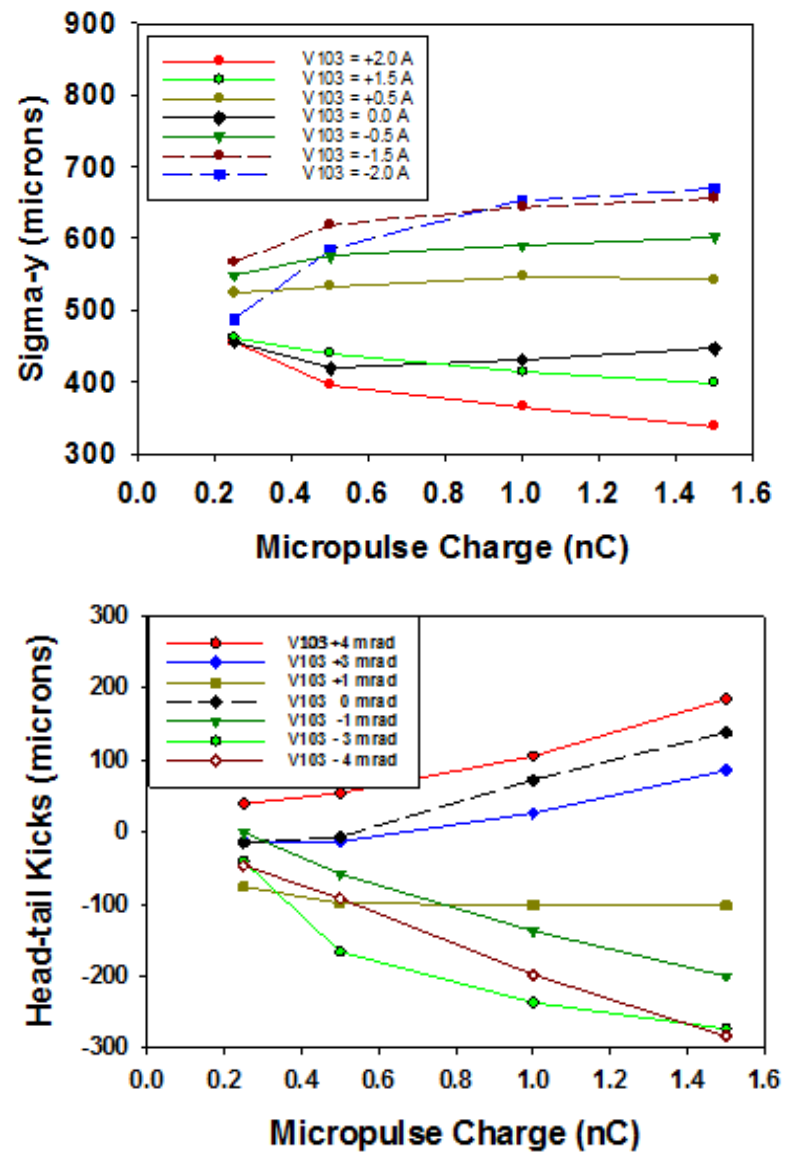

Figure 3: (a) Projected vertical beam profiles for X121 streak camera images versus charge at various V103 corrector values. (b) Observed head-tail centroid kicks for the same images as a function of charge and V103 corrector values. A 1-A corrector current change corresponds to a 2-mrad angular steering change into CC2. 
I data exhibit the direction the beam is off axis while the $\because \mathrm{HOMs}$ with the electronics used indicate only the offset magnitude. These combined effects may be used in a future machine learning training application to mitigate emittance dilution effects.

\%

\section{Space-Charge-Driven Bunch Elongation Effects}

The streak images when projected to the time axis provide the electron bunch profile and length. With an initial

Elaser pulse length of $4 \mathrm{ps}$, we observed the significant

क्षेectron bunch elongation due to space-charge forces as seen in Fig. 4. In this case the laser spot size was 票 $\sim 0.45 \mathrm{~mm}$ by $0.56 \mathrm{~mm}$ so at $250 \mathrm{pC} / \mathrm{b}$ and above the space-charge forces dominated the distributions.

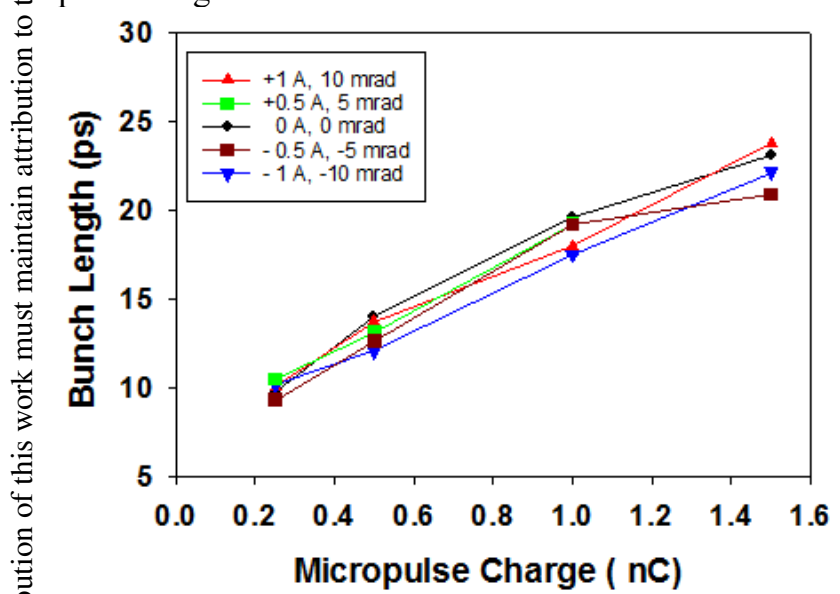

Figure 4: Streak camera bunch length versus charge results during the V101 corrector scan from -1.0- to +1.0-A values. At $4.5 \mathrm{MeV}$, a 1-A current change corresponds to $\dot{\sigma}$ a 10-mrad angular change into $\mathrm{CC} 1$. The transverse laser त्रे spot is $\sim 0.5 \mathrm{~mm}$ in $\mathrm{x}$ and $\mathrm{y}$ in this case.

\section{Bunch by Bunch RF BPM Data}

We also evaluated the potential long-range wakefield or HOM effects on the beam centroid by using the rf BPM $\dot{m}$ data. An example of the centroid motion within the 50- micropulse train of a macropulse is shown in Fig. 5 0 with both noise-reduction and bunch-by-bunch capabilio ties implemented. The $100-\mathrm{kHz}$ oscillation seen in the B117 data is a difference frequency between HOM mode 14 in CC2 and a beam harmonic [4]. The field oscillations E्] kicked different micropulses according to the amplitude at o that point in time, resulting in the submacropulse effects. The quadrupole triplet Q118-120 was used to focus the E beam smaller horizontally while leaving the vertical size $\Xi$ at $\sim 800 \mu \mathrm{m}$ at the X121 YAG:Ce station. The beam cen$\underset{g}{g}$ troid oscillation amplitude is much reduced to $<20 \mu \mathrm{m}$ at $\therefore$ B121 downstream of this triplet, and thus the main comDeting mechanism identified does not account for the observed $y$-t effect in the streak image. Since the ASTRA simulation for the FAST setup indicated a $400-\mu \mathrm{m}$ headtail kick for $500 \mathrm{pC} / \mathrm{b}$ and a $5-\mathrm{mm}$ offset at $41 \mathrm{MeV}$, we attribute the $100-\mu \mathrm{m}$ head-tail effects for a $2-3 \mathrm{~mm}$ offset seen in Fig. 3 to such short-range wakefields [11].
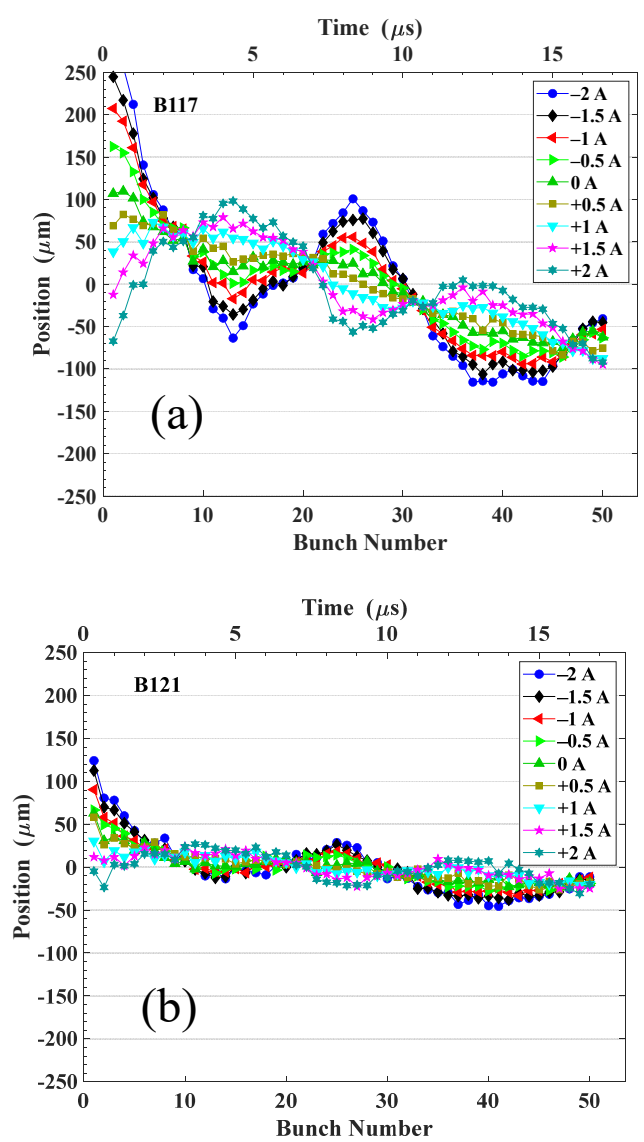

Figure 5: Examples of the variation of the beam vertical centroids bunch by bunch for 50 micropulses at B117 (a) and $\mathrm{B} 121$ (b) for $\mathrm{V} 103$ settings of $\pm 2 \mathrm{~A}$ from the reference. These were 100 -shot averages to show the $100-\mathrm{kHz}$ oscillation effects generated in CC2.

\section{ASTRA Simulations of the Short-range Wake- field}

The ASTRA simulations used the nominal FAST linac input deck with the laser bunch length of 4 ps sigma, a laser transverse spot size of $1.2 \mathrm{~mm}$ in $\mathrm{x}$ and $\mathrm{y}$, an injection phase of $45 \mathrm{o}$, a gun gradient of $45 \mathrm{MV} / \mathrm{m}$, and the capture cavity gradients of 21 and $14 \mathrm{MV} / \mathrm{m}$ for $\mathrm{CC} 1$ and $\mathrm{CC} 2$, respectively. In particular, the program utilizes the 3D fields of the TESLA-type cavities to produce realistic simulations of the beam through the cavities for comparison to the observations. The simulations were for a single micropulse with $500 \mathrm{pC}$ of charge transiting the two cavities with $0 \mathrm{~mm}$ and $5 \mathrm{~mm}$ offsets as shown in Fig. 6 .

The time-sliced vertical distributions at the head and tail of the micropulse are shown. The simulation shows a tear-drop shape in y-t space (envision later time upward) as reported previously [5] and an approximately $400-\mu \mathrm{m}$ head-tail kick for the 5-mm offset in Fig. 6 b.

In Fig. 7 the elongation of the electron bunch is calculated as $\sigma_{t}=11.2 \mathrm{ps}$ compared to the 4-ps laser pulse. This is attributed to space-charge forces acting before acceleration in $\mathrm{CC} 1$. The charge dependence of the bunch length 

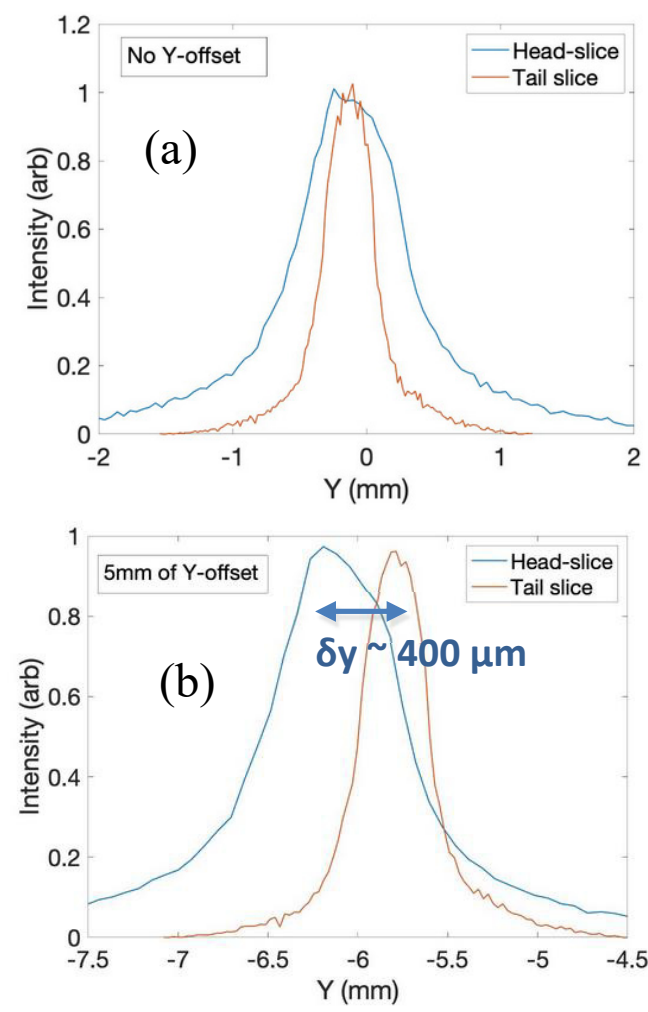

Figure 6: ASTRA simulation results of the head-tail kick for (a) no y offset and (b) 5-mm y offset through the cavities with a calculated $\sim 400-\mu \mathrm{m}$ head-tail kick.

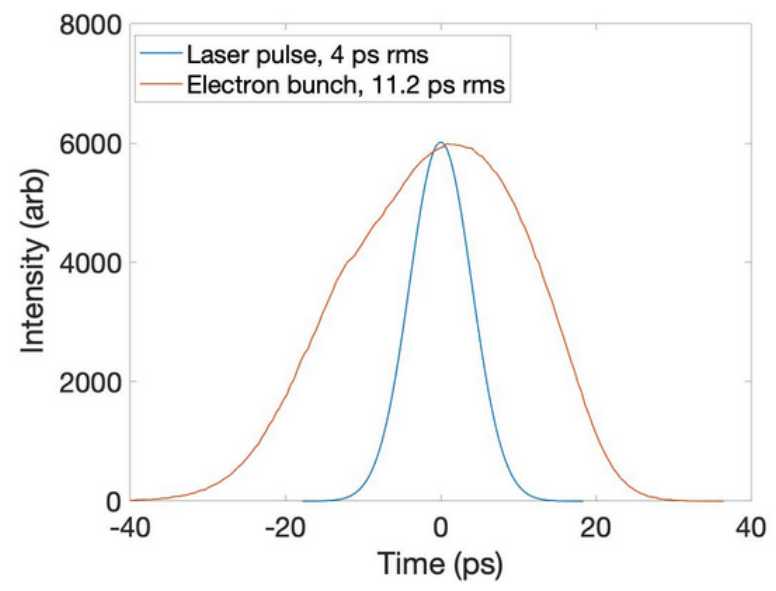

Figure 7: ASTRA simulation results for the electron-beam bunch length at $500 \mathrm{pC} / \mathrm{b}$ compared to the initial laser pulse length.

from ASTRA is shown in Fig. 8 for the laser transverse spot size of $1.2 \mathrm{~mm}$ and the initial laser pulse of 4-ps duration. The electron beam rms bunch length varies from $\sim 10$ to $14 \mathrm{ps}$ for 0.25 to $1.5 \mathrm{nC} / \mathrm{b}$, respectively. There would be an expected increase in this effect for a smaller laser spot size of $0.5 \mathrm{~mm}$ as seen in the Fig. 4 data.

\section{SUMMARY}

In summary, observations of short-range wakefield effects on beam dynamics were made using the streak camera to obtain $y-t$ images at the submicropulse time scale.

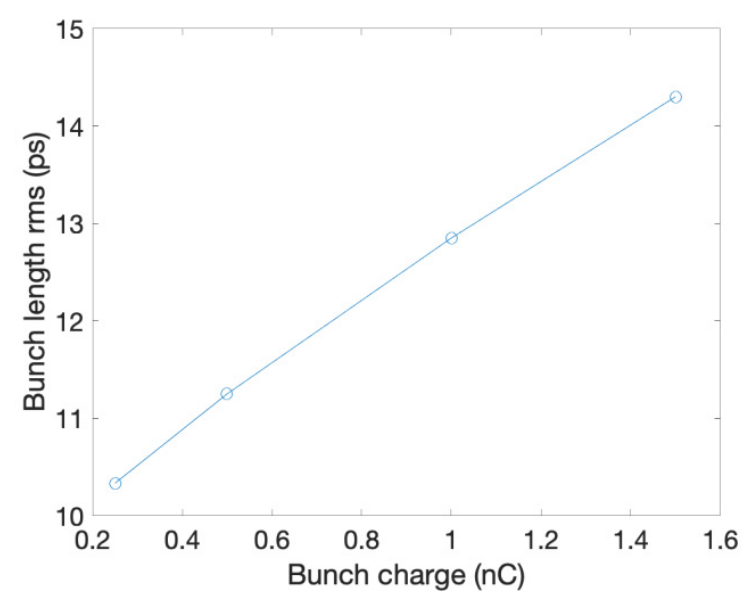

Figure 8: ASTRA simulation results for the charge dependence of the observed bunch length after $\mathrm{CC} 2$. The laser pulse length was $4 \mathrm{ps}$.

The HOM detectors and rf BPMs were used to evaluate off-axis steering related to these tests, and the HOMinduced sub-macropulse centroid motion was shown to be much smaller than the observed effects. Moreover, the head-tail centroid kicks were consistent with short-range wakefield results from ASTRA for the TESLA-type superconducting rf cavity and attributed to that effect.

\section{ACKNOWLEDGMENTS}

The Fermilab authors acknowledge the support of $\mathrm{C}$. Drennan, A. Valishev, D. Broemmelsiek, G. Stancari, and M. Lindgren, all in the Accelerator Division at Fermilab. The SLAC/NAL authors acknowledge the support of J. Schmerge (Superconducting Linac Division, SLAC).

\section{REFERENCES}

[1] H. Weise, "Commissioning and First Lasing of the European XFEL", in Proc. FEL'17, Santa Fe, NM, USA, Aug. 2017, pp. 9-13. doi:10.18429/JACoW-FEL2017-MOCO3

[2] P. Emma, "Status of the LCLS-II FEL Project at SLAC", presented at the 38th Int. Free Electron Laser Conf. (FEL'17), Santa Fe, NM, USA, Aug. 2017, paper MOD01, unpublished.

[3] J. T. Seeman et al., "Transverse wakefield control and feedback in the SLC linac", SLAC National Accelerator Laboratory Rep. SLAC-Pub-4182, 1987.

[4] A. H. Lumpkin et al., "Submacropulse electron-beam dynamics correlated with higher-order modes in Tesla-type superconducting rf cavities", Phys. Rev. Accel. and Beams vol. 21, p. 064401, 2018. doi : 10.1103/PhysRevAccelBeams . 21.064401

[5] A.H. Lumpkin, R.M. Thurman-Keup, D. Edstrom, and J. Ruan, "Submicropulse electron-beam dynamics correlated with short-range wakefields in Tesla-type superconducting rf cavities", Phys. Rev. Accel. and Beams, vol. 23, p. 054401 , doi:10.1103/PhysRevAccelBeams . 23 . 054401 2020.

[6] P. H. Garbincius et al., "The ASTA User Facility Proposal", FNAL, Batavia, IL, USA, Rep. Fermilab-TM-2568, October 2013.

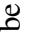
胥

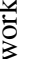
包 
[7] J. Ruan, M. D. Church, D. R. Edstrom, T. R. Johnson, and J. K. Santucci, "Commission of the Drive Laser System for Advanced Superconducting Test Accelerator", in Proc. IPAC'13, Shanghai, China, May 2013, paper WEPME057, pp. 3061-3063.

[8] R. Thurman-Keup, FNAL, off-line MATLAB-based ImageTool, 2011.

[9] A. H. Lumpkin, J. Ruan, and R. Thurman-Keup, "Synchroscan streak camera imaging at a $15-\mathrm{MeV}$ photoinjector with emittance exchange", Nucl. Instrum. Methods Phys. Res., Sect. A, vol. 687, pp. 92-100, 2012. doi:10.1016/j.nima.2012.05.068

[10] A. H. Lumpkin, D. Edstrom, and J. Ruan, "Initial Demonstration of 9-MHz Framing Camera Rates", in Proc. NAPAC16, Chicago, IL, USA, Oct. 2016, pp. 333-336 doi : 10. 18429/JACoW-NAPAC2016-TUPOA25

[11] V. Lebedev, private communication, May 2016. 